\title{
3D MODELING OF RECYCLING EQUIPMENT BY MAGNETIC SEPARATION OF USED SANDBLASTING SAND
}

\author{
Dorin EFTIMIE \\ "Dunarea de Jos" University of Galati, Romania \\ e-mail: dorin.eftimie@ugal.ro
}

\begin{abstract}
The paper presents the $3 D$ modelling of recycling equipment by magnetic separation of the used sandblasting sand. The modelling was done using the software NX 7.5 from Siemens.

Magnetic separation is performed in three stages, two steps in dry separation and one step in wet separation. The magnetic separation operation is performed with three drums with remnant magnets designed optimized according to the design requirements.

$3 D$ modelling of this type of equipment will allow modular design for any parameters required by the design theme.
\end{abstract}

KEYWORDS: recycling used sandblasting sand, 3D modelling of recycling equipment

\section{Overview}

Sandblasting is the process of cleaning or finishing by abrasive blasting of surfaces made of metal, stone, glass or other solid material. This is done with the help of metallic pellets, sand or other granular abrasive materials which are driven with speed by mechanical centrifugation or by a jet of gas (compressed air) or liquids (pressurized water) to the surfaces to be processed. Sandblasting is used when, in order to paint, it is necessary to clean complex metal surfaces that do not allow the use of other methods. It is executed in enclosed spaces (Fig. 1) due to the high degree of danger presented by the jet of compressed air and sand at very high speeds [1].

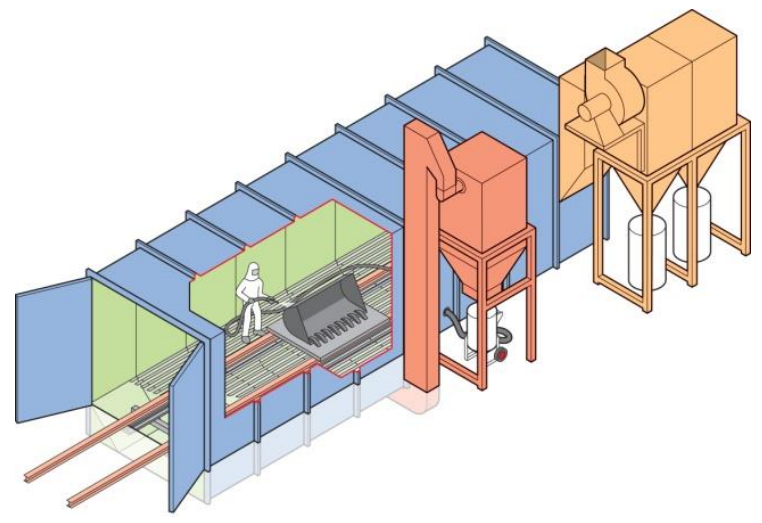

Fig. 1. Sandblasting chamber
Sandblasting is used in the metallurgical industry, shipyards and generally speaking wherever rapid cleaning of surfaces is required.

The sand is used for sandblasting, because it is very cheap, but for environmental and operator protection reasons, its use has been restricted.

\section{3D modelling of recycling equipment}

Figure 2 shows the principle of separation of the metal sand mixture by means of a magnetic separation system. The 3D modelling was made with NX 8.0 software of Siemens. The metal sand mixture is introduced into the bowl. Using a conveyor belt, it is transported to the magnetic separator. The magnetic separator with three magnet drums separates the metal sand. As shown in Figure 2 the drums have the opposite direction of the clockwise. The first separation that takes place in the first drum is a dry separation. After the mixture passes through the first drum, a metallic part runs on the drum and falls gravitationally into the separation of metallic particles. The second separation is made even in the second drum. The metal particles in the mixture stick to the drum and fall gravitationally into the separation of metal particles. The last drum makes a wet magnetic separation. Sand and water enter into fine sites where the water separates from the sand. The water is transported by means of pumps to a filtration unit, which is then fed back into the system. 

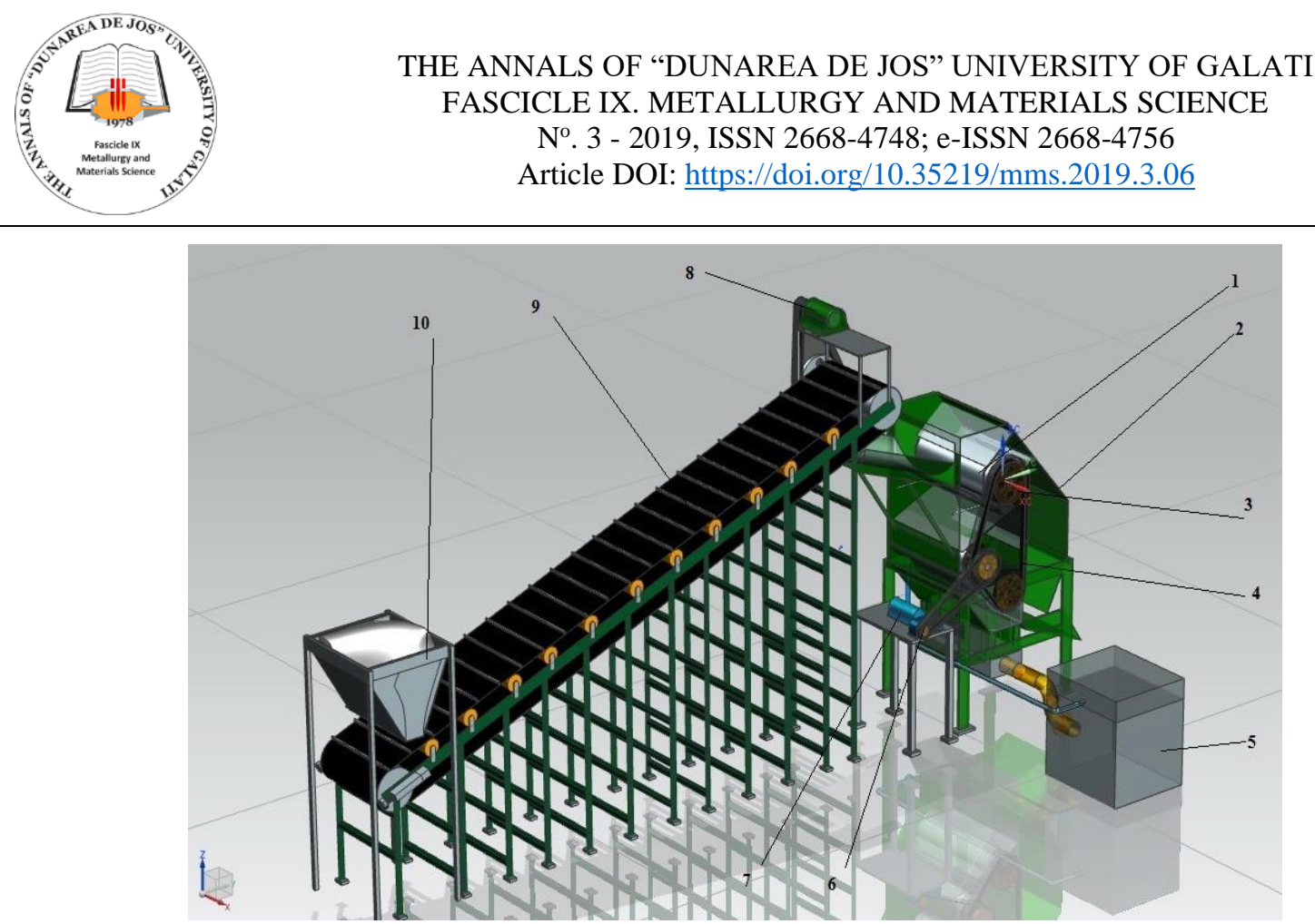

Fig. 2. The general assembly of the magnetic separation system of the sandblasted sand 1 - Drum with permanent magnets, 2 - framework of the magnet separator, 3 - belt drive, 4 - belt, 5 water purification unit, 6 - reducer, 7 - electric motor for driving the drums, 8 - electric motor for driving the conveyor belt with rake, 9 - conveyor belt with racks, 10 - tank with sandblasted sand

The first subassembly that was brought in the working window is the conveyor belt with racks. As shown in Figure 2, the conveyor belt is composed of the fixed frame which the rollers are mounted. The tape is made of a rubber material and is driven by two drums, the driving drum and the driven drum. The driving drum is driven by an electric motor with variable speed. The speed of the driving drum must be correlated with the speed of the magnetic separation drums. The transmission ratio from the electric motor shaft to the drive drum drive wheel is 1:5. The driven drum slides on a rail to extend the conveyor belt with two tension straps.

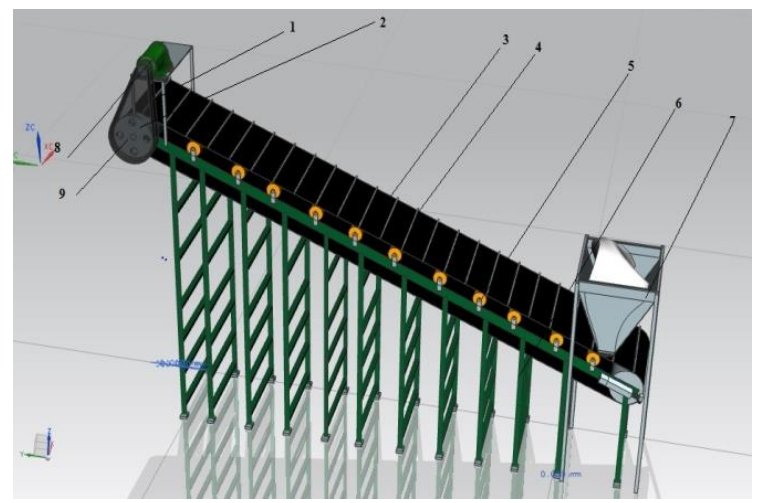

Fig. 3. Conveyor belt with racks 1 - drive belt, 2 - drive drum wheel, 3 - belt, 4 - rake, 5 - roller, 6 - rigid metal frame, 7 - tank, 8 - electric drum drive motor, 9 - belt guard
In Figure 3 you can see the conveyor belt with the main components that compose it.

The magnetic separator is the most important subassembly in the magnetic separation system. This can be seen in Figure 4. As seen, it is driven by an electric motor. The speed of magnetic separation drums is reduced by means of a reducer.

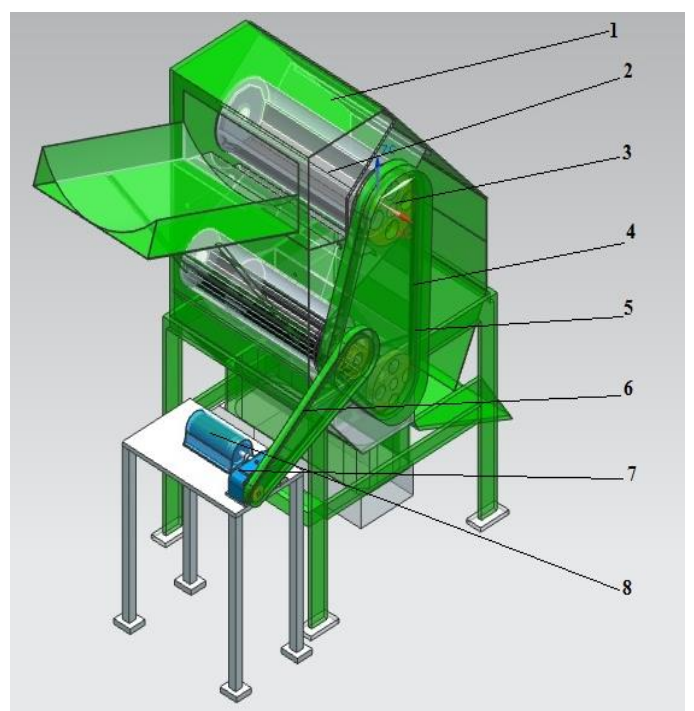

Fig. 4. Magnetic separator 1 - Framework, 2 magnetic drum, 3 - belt wheel, 4 - belt guard, 5 drum drive belt, 6- reducer drive belt- magnetic separator, 7-reducer, 8- magnetic separator drive motor 


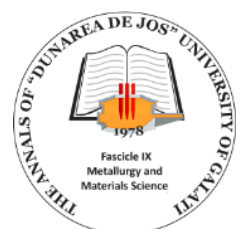

As shown in Figure 5, the magnetic separator with permanent magnets has three magnetic reels which are driven by a wide belt. The last drum of the magnetic separator is sprinkled with a splash. The sprinkler is a pipe with a diameter of $\mathrm{mm}=20 \mathrm{~mm}$ and has holes of $5 \mathrm{~mm}$ in diameter, positioned at distances equal to $20 \mathrm{~mm}$. The last drum performs a wet separation. The sprinkler can be seen in figure 3, where we have a longitudinal section of the magnetic separator.

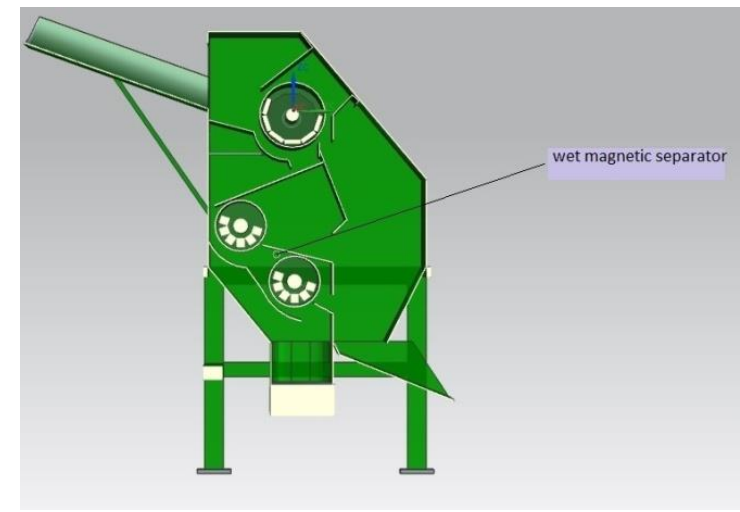

Fig. 5. Magnetic separator section

The main subassembly of the magnetic separator is the permanent magnet drum see Figure 6.

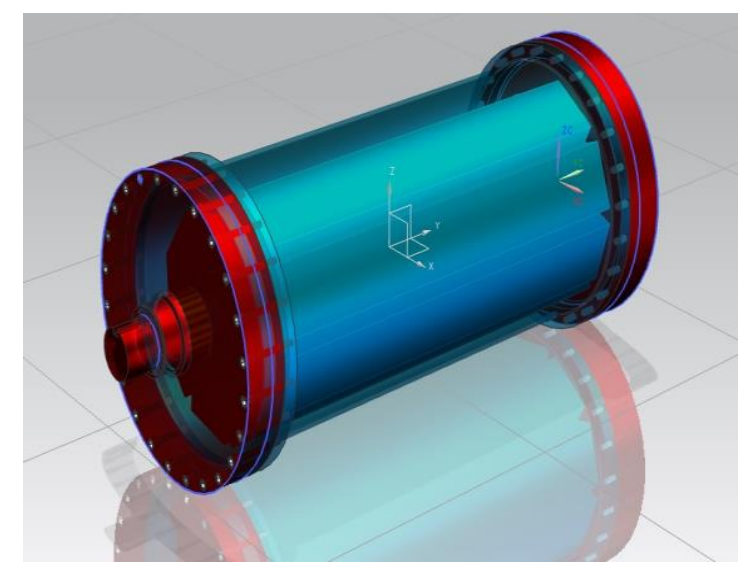

Fig. 6. Drum with permanent magnets

The permanent magnet drum is a subassembly made up of a plate with 2 welded shoulders (see Figure 7) at the ends to attach to two ends caps.

The cover that covers the screwed sheet is clamped with hex screws with hexagon head. The cover must be mechanically machined because it also acts as a drive shaft for the drum.

The cover can be seen in Figure 8. As seen, it is provided with a wedge channel for fastening the belt wheel.

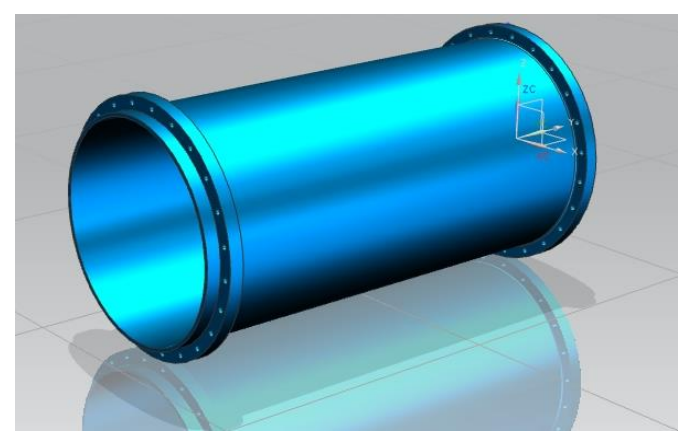

Fig. 7. Drum housing

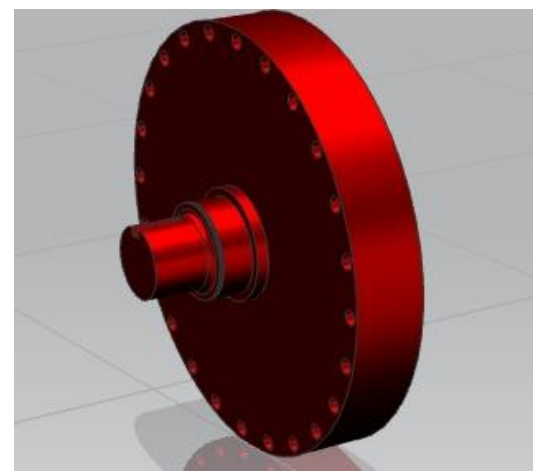

Fig. 8. Drum cover

Inside the drum housing there is a subassembly formed of two polygonal plates having 10 sides each and on which six permanent magnets are glued. Polygonal plates are pierced by a shaft, and caught in mounting bolts by the shaft flanges. The shaft is machined at the end as it enters these two bearings with needles. A Seeger ring is provided as the end of the bearings. The arrangement of the magnets can be seen in the subassembly of Figure 9.

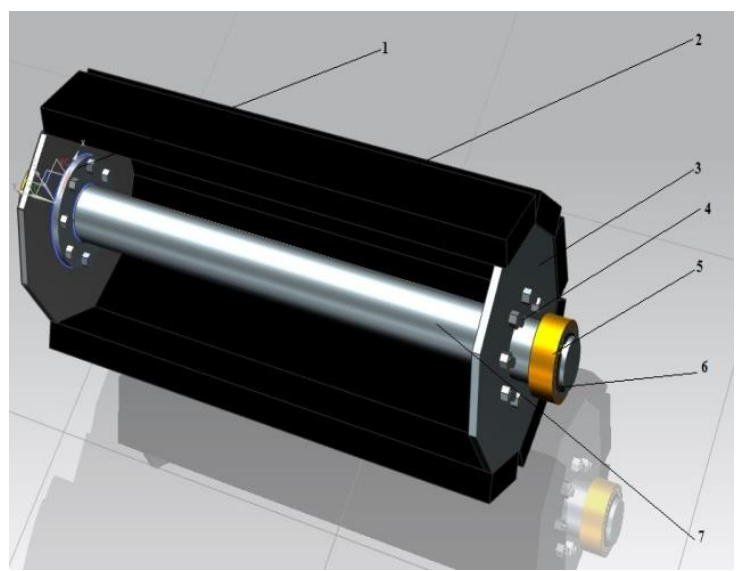

Fig. 9. The arrangement of permanent magnets 1 - Welded flange, 2 - permanent magnets, 3 Polygonal plate, 4 - fixing screws, 5 - needle bearing, 6 - Seeger ring, 7 - axis 

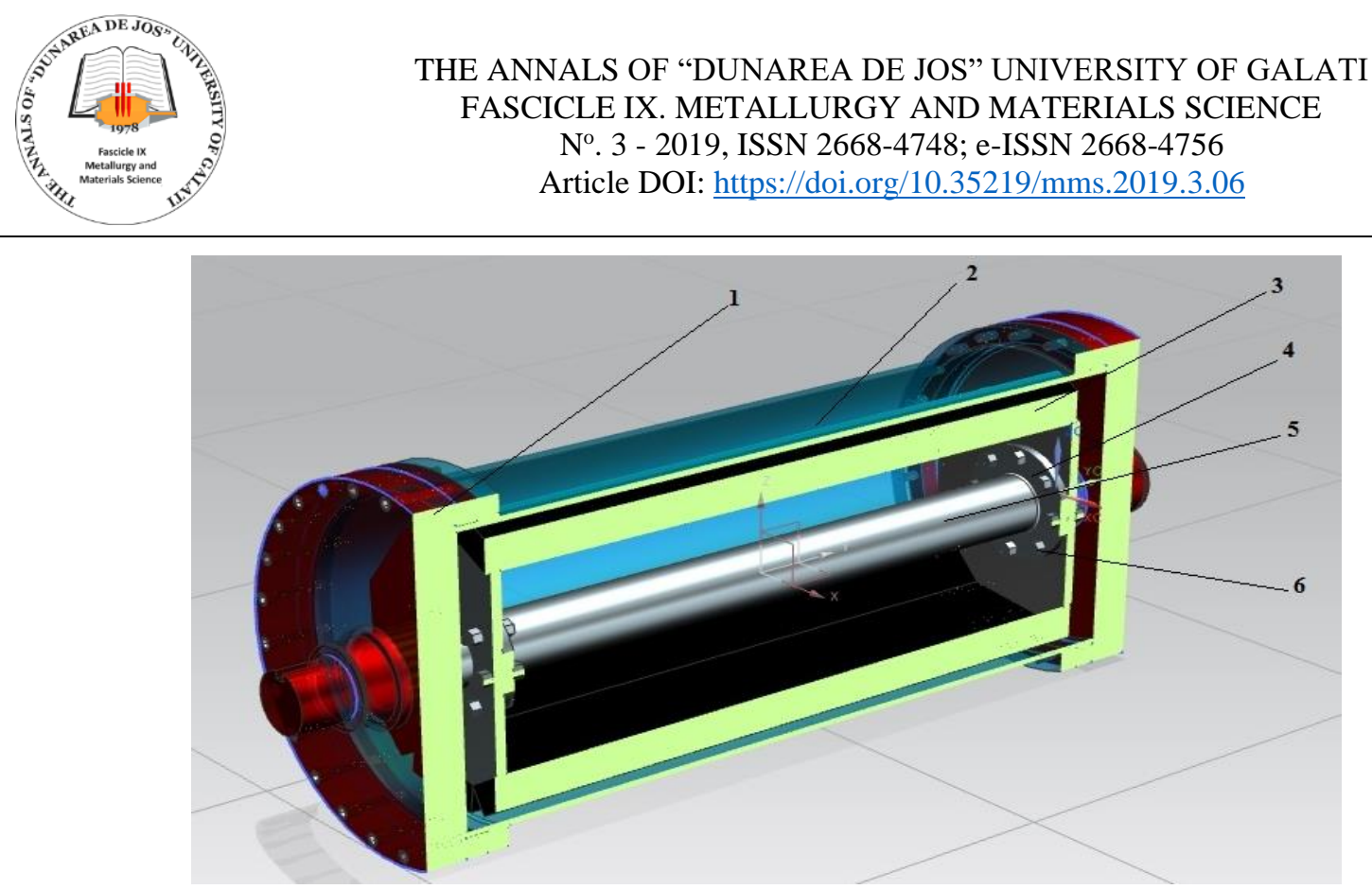

Fig. 10. Magnetic drum section 1 - Drum cover, 2 - drum housing, 3 - magnets, 4 - welded flange, 5 fastening screws

The subassembly is mounted in the covers provided with mounting bearings. What is interesting about this drum is that the drum has a rotating motion, and the permanent magnets remain in a resting position. This is due to bearings that have a rolling motion and reduced friction between the cover port and the magnet axis. For a clearer image of mounting the subassembly, a section was made through it in Figure 10.

\section{Conclusions}

After the blasting process, the following wastes appear in the EC Framework Directive no. 75/442/EEC:

- wastes from the removal of paints and varnishes containing organic solvents or other dangerous substances;
- wastes in the form of powders in suspension which occur especially when blasting with air;

- wastes resulting from cleaning of metallic surfaces of rust and shear, especially encountered on surfaces that are painted first;

- waste resulting in the form of dust and suspensions of ferrous metals;

- waste from welding operations;

- wastes from blasting material.

The sand used in the blasting operation is found in large quantities at the shipyards and is an environmental problem. The equipment proposed in the paper represents an ecological solution within the line of recycling of the used sand used for blasting.

\section{References}

[1]. ***, https://www.worksafe.qld.gov.au [2]. ***, High-End solution CAD/CAM/CAE Siemens NX 8.0 